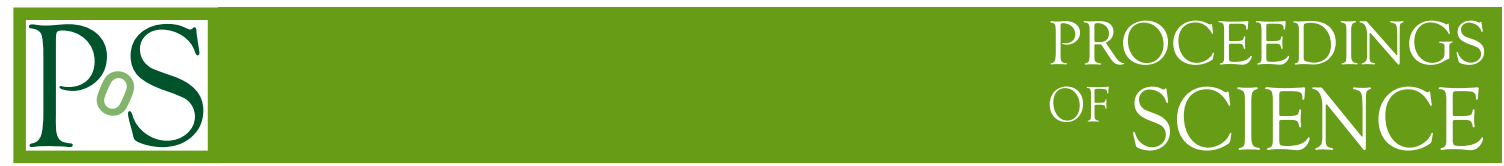

\title{
Searches for non-resonant $\mathrm{HH}$ production at CMS
}

\author{
Alessandra Cappati ${ }^{* \dagger}$ \\ University and INFN Torino, Italy \\ E-mail: alessandra.cappati@cern.ch
}

The most recent results from searches for non-resonant production of Higgs boson pairs (HH) at CMS are presented. Four final states have been studied, with data collected at $\sqrt{s}=13 \mathrm{TeV}$ in 2016, corresponding to an integrated luminosity of $35.9 \mathrm{fb}^{-1}$. The combination of the four channels can set an observed (expected) upper limit on the HH production cross section to 22.2 (12.8) times the SM prediction. Beyond the SM scenarios for HH production are also explored.

European Physical Society Conference on High Energy Physics - EPS-HEP2019 -

10-17 July, 2019

Ghent, Belgium

* Speaker.

${ }^{\dagger}$ on behalf of the CMS Collaboration 


\section{The double-Higgs boson non-resonant production}

The non-resonant double-Higgs boson (HH) production can be used to directly study the Higgs boson (H) self coupling. At the CERN Large Hadron Collider (LHC), HH is mainly produced through the gluon-gluon fusion mechanism via a fermion loop, but accordingly to the Standard Model (SM) of particle physics there is a destructive interference between the diagram with triangle fermion loop and the box contribution (Fig. 1, top part). This results in a small HH production cross section.

Beyond Standard Model (BSM) processes could modify the cross section and the kinematic properties of the $\mathrm{HH}$ production process. This scenario is treated with an Effective Field Theory (EFT) approach that results in 5 parameters that control the tree-level interactions (see Fig. 1). Two of them express the deviation from the SM: $k_{\lambda}$, defined as the trilinear coupling of the Higgs boson over the SM expectation for this coupling, and $k_{t}$, which is the Yukawa coupling of the top quark over the SM expectation. The other 3 parameters represent additional BSM couplings: $c_{2}$, defined as the coupling between 2 top quarks and 2 Higgs bosons, $c_{g}$, coupling between 2 gluons and one Higgs boson, and $c_{2 g}$, coupling between 2 gluons and 2 Higgs bosons. In order to study the different possible modifications of the SM double-Higgs boson signal, 12 benchmarks have been defined varying the values of these 5 parameters [1].

At the CERN LHC a rich phenomenology is accessible to study the double-Higgs boson process. The CMS Collaboration [2] performed HH searches already at the LHC Run 1, studying the $b \bar{b} \tau \tau$ and $b \bar{b} \gamma \gamma$ final states, and the combined upper limit set on HH production was of 43 times the SM expectation. In the LHC Run 2 there are new opportunities for HH searches thanks to the increase of the production cross section and to the accessibility of new decay channels. Up to now the CMS Collaboration explored 4 different final states (b $\bar{b} b \bar{b}, b \bar{b} 2 \ell 2 v, b \bar{b} \tau \tau$ and $b \bar{b} \gamma \gamma)$ using data collected in 2016, corresponding to an integrated luminosity of $35.9 \mathrm{fb}^{-1}$, and a combination was performed with the results obtained.
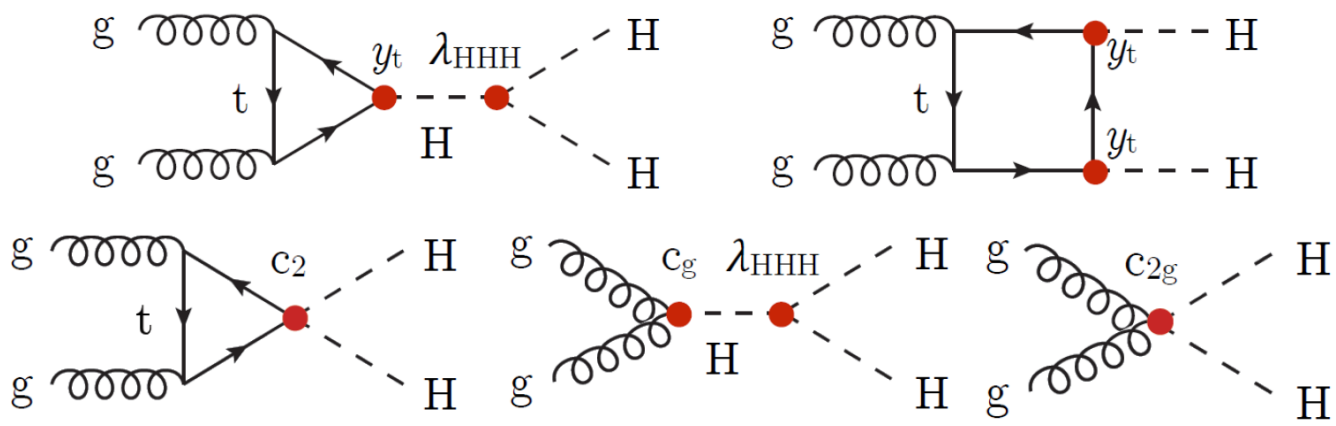

Figure 1: Feynman diagrams that contribute to HH production via gluon-gluon fusion at LO. The two top diagrams correspond to SM-like processes, while the three bottom diagrams correspond to pure BSM effects: the first one describes the contact interaction of two Higgs bosons with two top quarks, while the second and the third ones describe contact interaction between two gluons and one or two Higgs bosons, respectively [1]. 


\section{2. $\mathrm{HH} \rightarrow \mathrm{b} \bar{b} b \bar{b}$}

For the searches of double-Higgs boson in the final state with $4 \mathrm{~b}$ jets [3], events are selected if there are 4 identified $b$ jets, that then are paired to form Higgs boson candidate. A Boosted Decision Tree (BDT) is trained to distinguish the SM signal from backgrounds and enhance the sensitivity of the analysis. The background is estimated from data with an hemisphere mixing technique, which consists in dividing each data event in two hemispheres and then the collection of hemispheres is used to create new data-like events by recombining hemispheres in pairs. The dominant background source consists of QCD multi-jet events. A likelihood fit is performed on the BDT distribution in order to extract the results. No excess is observed in data, therefore an upper limit is set on the cross section for $\mathrm{pp} \rightarrow \mathrm{HH} \rightarrow \mathrm{b} \overline{\mathrm{b}} \overline{\mathrm{b}}$ non-resonant production. The expected $95 \%$ confidence level (CL) limit is $419 \mathrm{fb}$, which corresponds to 37 times the SM HH production cross section times the square of the branching fraction for the $\mathrm{H} \rightarrow \mathrm{b} \bar{b}$ decay. The observed upper limit obtained is $847 \mathrm{fb}$, which is 2 standard deviations above the expected upper limit.

Searches of BSM HH production with values of $k_{\lambda}$ in the range [-20,20], assuming $k_{t}=1$, are performed. Results are shown in Fig. 2 (left). No value of $k_{\lambda}$ is excluded.

\section{3. $\mathrm{HH} \rightarrow \mathrm{b} \overline{\mathrm{b}} 2 \ell 2 v$}

For searches of $\mathrm{HH}$ production in the $\mathrm{b} \overline{\mathrm{b}} 2 \ell 2 \mathrm{v}$ final state [4], events are selected when there are 2 opposite sign leptons and $2 \mathrm{~b}$ jets. The events selected can come both from $\mathrm{b} \overline{\mathrm{b}} Z \mathrm{Z} \rightarrow \mathrm{b} \overline{\mathrm{b}} \ell \boldsymbol{v} \ell v$ and $\mathrm{b} \overline{\mathrm{b}} \mathrm{WW} \rightarrow \mathrm{b} \overline{\mathrm{b}} v v \ell \ell$ processes, but a mass requirement $\left(12<m_{\ell \ell}<m_{Z}-15 \mathrm{GeV}\right)$ is set to suppress quarkonia resonances and $\mathrm{Z}$ boson background. This channel is characterized by a large irreducible
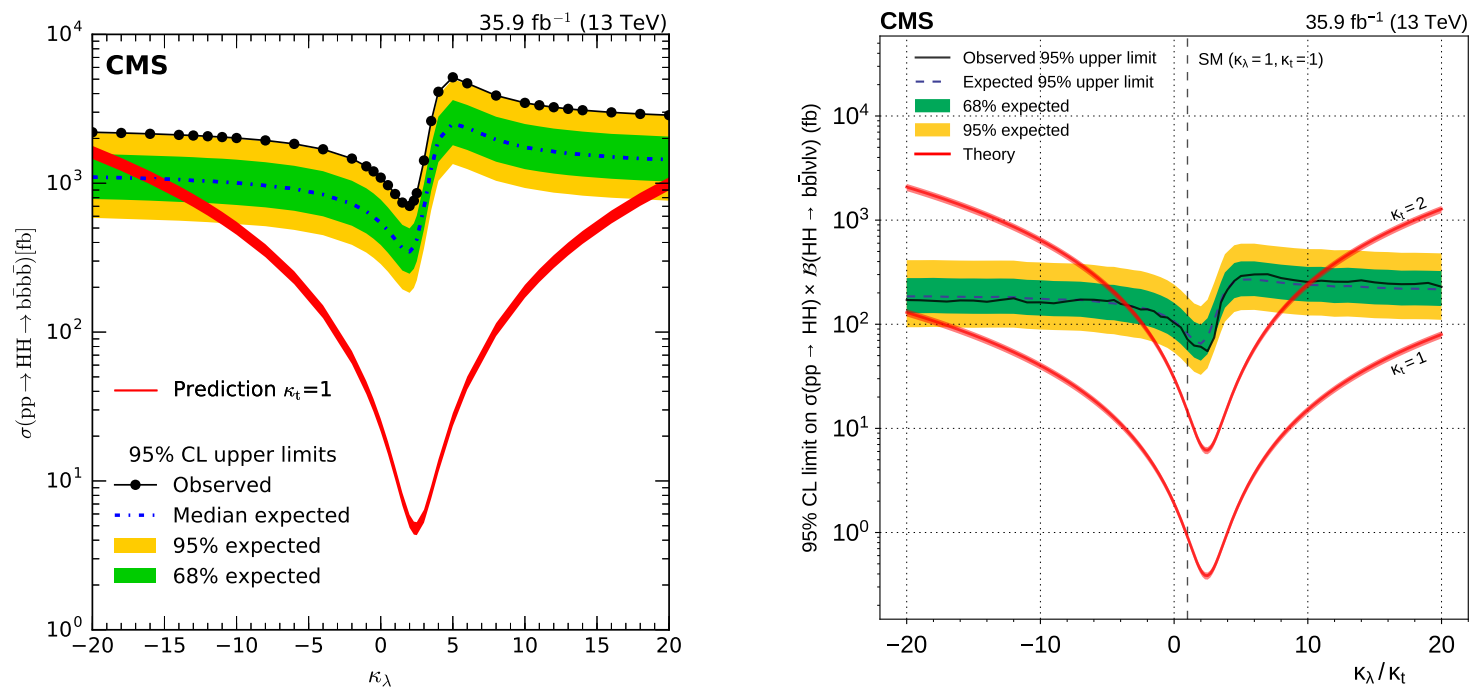

Figure 2: Left: $95 \% \mathrm{CL}$ limits on $\sigma(\mathrm{pp} \rightarrow \mathrm{HH} \rightarrow \mathrm{b} \bar{b} \mathrm{~b} \bar{b})$ for values of $k_{\lambda}$ in the [-20,20] range, assuming $k_{t}=1$; the theoretical prediction with $k_{t}=1$ is also shown [3]. Right: $95 \%$ CL limits on $\sigma$ (pp $\rightarrow \mathrm{HH} \rightarrow$ $\mathrm{b} \overline{\mathrm{b}} \ell v \ell v)$, as a function of $k_{\lambda} / k_{t}$; red lines show the theoretical cross sections, along with their uncertainties, for $k_{t}=1(\mathrm{SM})$ and $k_{t}=2[4]$. 
background due to $t \bar{t}$ and Drell-Yan (DY) events, therefore a Deep Neural Network (DNN) is trained to discriminate between signal and background. A binned Maximum Likelihood (ML) fit is then performed over the DNN distributions. No evidence for $\mathrm{HH}$ production is observed in data, thus an observed upper limit is set on the SM HH $\rightarrow \mathrm{b} \overline{\mathrm{b} V V} \rightarrow \mathrm{b} \overline{\mathrm{b}} \ell v \ell v$ cross section to $72 \mathrm{fb}$, in agreement with an expected upper limit of $81 \mathrm{fb}$. The observed upper limit amounts to 79 times the SM prediction, in agreement with an expected upper limit of 89 times the SM prediction.

In the BSM searches, limits are set as a function of $k_{\lambda} / k_{t}$ with 2 different hypotheses for $k_{t}$, as shown in Fig. 2 (right).

\section{4. $\mathrm{HH} \rightarrow \mathrm{b} \bar{b} \tau \tau$}

For searches of HH production in the $b \bar{b} \tau \tau$ final state [5], events are selected if there is one isolated $\tau$ lepton decaying hadronically and a second opposite charge lepton; then events are categorized according to the number of $\mathrm{b}$ jets. The main background contributions come from $\mathrm{t} \overline{\mathrm{t}}$, DY and multi-jet events. A BDT is trained in order to reduce the tit background contribution. The stranverse mass variable is found to provide the best signal to background separation. This variable is defined as the largest mass of the parent particle that is compatible with the kinematic constraints of the event: in the case of the $b \bar{b} \tau \tau$ decay, where the dominant background is tit production, the parent particle is interpreted as the top quark that decays into a bottom quark and a $\mathrm{W}$ boson. The presence of non-resonant $\mathrm{HH}$ production would result in an enhancement in the tail of the stranverse mass distribution. A binned ML fit is performed on the stranverse mass distribution; no evidence of $\mathrm{HH}$ production is observed in data, thus an upper limit is set on the cross section times branching fraction. The observed 95\% CL limit amounts to $75.4 \mathrm{fb}$, while the expected 95\% CL limit is 61.0 fb. These values correspond to about 30 and 25 times the SM prediction, respectively.

For BSM HH production searches, limits are set for different hypotheses of anomalous selfcoupling and top quark coupling of the Higgs boson. Limits are set as a function of $k_{\lambda} / k_{t}$, under
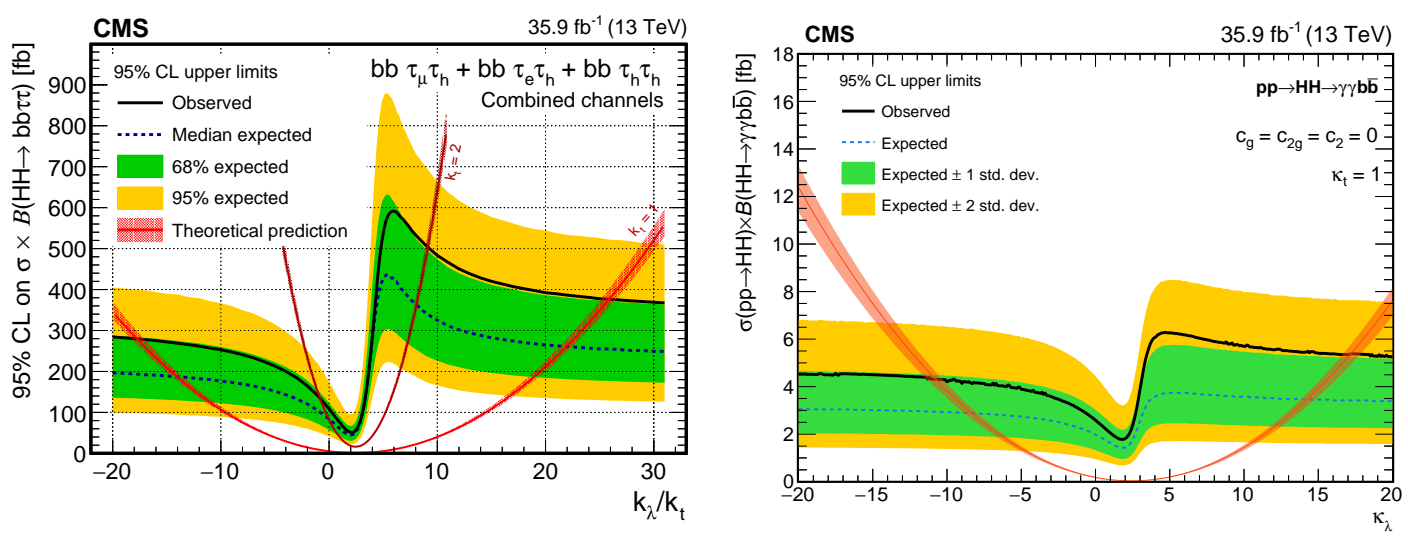

Figure 3: Left: Observed and expected 95\% CL upper limits on $\sigma(\mathrm{pp} \rightarrow \mathrm{HH} \rightarrow \mathrm{b} \overline{\mathrm{b}} \tau \tau)$ as a function of $k_{\lambda} / k_{t}$; the two red bands show the theoretical cross section expectations and the corresponding uncertainties for $k_{t}=1$ and $k_{t}=2$ [5]. Right: Observed and expected 95\% CL upper limits on $\sigma(\mathrm{pp} \rightarrow \mathrm{HH} \rightarrow \mathrm{b} \bar{b} \gamma \gamma)$ as a function of $k_{\lambda}$; the red band shows the theoretical cross section expectations and the corresponding uncertainties for $k_{t}=1$ [6]. 
two hypotheses for $k_{t}$, as shown in the Fig. 3 (left).

\section{5. $\mathrm{HH} \rightarrow \mathrm{b} \bar{b} \gamma \gamma$}

For searches of HH production in the $b \bar{b} \gamma \gamma$ final state [6], events are selected if there are 2 identified photons and $2 \mathrm{~b}$ jets. The main background comes from events with $\gamma$ and jets, and it is estimated from mass side-bands. Events are classified into categories according to the $\mathrm{HH}$ reduced mass $\left(M_{x}=m_{j j \gamma \gamma}-\left(m_{j j}-m_{H}\right)-\left(m_{\gamma \gamma}-m_{H}\right)\right)$ and the event purity, which is estimated with BDT discriminants. A 2 dimensional fit is performed on the di-photon and di-jet invariant mass in order to extract the results. No evidence of $\mathrm{HH}$ production is observed in data, thus an upper limit is set on the cross section for $\mathrm{pp} \rightarrow \mathrm{HH} \rightarrow \mathrm{b} \overline{\mathrm{b}} \gamma \gamma$ non-resonant production. The observed 95\% CL upper limit amounts to $2 \mathrm{fb}$, while the expected is $1.6 \mathrm{fb}$. These values correspond to about 24 and 19 times the SM prediction, respectively.

For BSM HH searches, a scan on the coupling modifier $k_{\lambda}$ is performed in the range [-20,20], with $k_{t}=1 . k_{\lambda}$ values are constraint in the range $[-11,17]$ at $95 \% \mathrm{CL}$, as shown in Fig. 3 (right).

\section{Combination}

A combination of all the presented analyses was performed [7] and the results are presented in terms of signal strength modifier $\left(\mu=\sigma_{H H} / \sigma_{H H}^{S M}\right)$ on the HH production cross section in Fig. 4 (left). This plot shows the limit obtained in Run 1 and the combined limit from Run 2. For the latter, the observed signal strength is 22.2 while the expected is 12.8 . Results are compatible with expectations in all the channels. Only in the $4 \mathrm{~b}$ final state a tension is observed, but it is still compatible with the expected values. BSM HH production is searched with different values of the coupling modifier $k_{\lambda}$ while keeping the other EFT parameters fixed to their SM values. Exclusion limits
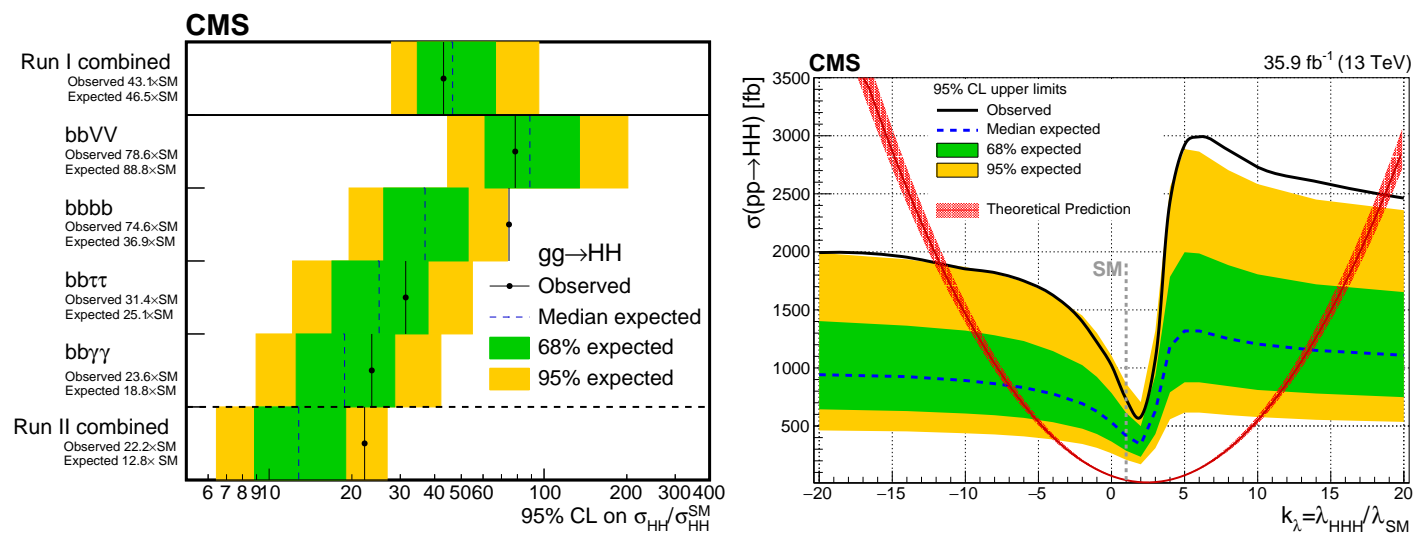

Figure 4: Left: The 95\% CL upper limits on the signal strength modifier $\mu=\sigma_{H H} / \sigma_{H H}^{S M}$. The inner (green) band and the outer (yellow) band indicate the regions containing 68 and 95\%, respectively, of the limits on $\mu$ expected under the background-only hypothesis [7]. Right: Expected and observed 95\% CL upper limits on the $\mathrm{HH}$ production cross section as a function of the $k_{\lambda}$ parameter. The red band shows the expected theoretical cross section expectations and its uncertainty. All other couplings and EFT parameters are set to their SM values [7]. 


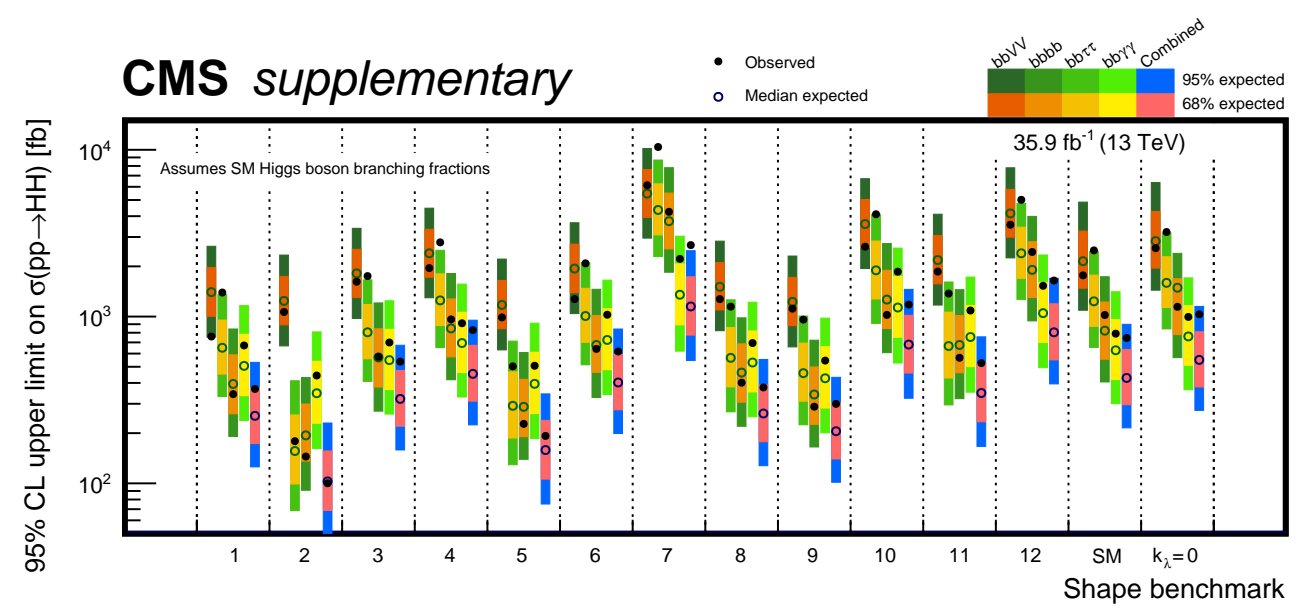

Figure 5: The 95\% CL exclusion limits on the non-resonant Higgs boson pair production cross sections for different EFT benchmark topologies, as defined in Ref. [1]. Each benchmark represents a possible modification in the $\mathrm{HH}$ signal yield and kinematic distributions due to BSM effects. The last two bins show the $95 \%$ CL exclusion limits for the SM and for the $k_{\lambda}=0$ scenarios. Limits are shown for each of the four final states separately and for the combination [7].

are set as function of $k_{\lambda}$, as shown in Fig. 4 (right). The $k_{\lambda}$ parameter is observed (expected) to be constrained to the range $-11.8<k_{\lambda}<18.8\left(-7.1<k_{\lambda}<13.6\right)$. The BSM searches are also performed in the 12 different EFT benchmarks setting upper limit on the HH production cross section. Fig. 5 shows the results obtained in the 4 different final states and for the combination of these results. Each benchmark represents a possible modification in the $\mathrm{HH}$ signal yield and in the kinematic decay due to BSM effects. The last 2 bins of the plot show the exclusion limits (at 95\% $\mathrm{CL}$ ) for the SM scenario and the hypothesis where the trilinear coupling is 0 . All results are found to be compatible with expectations.

\section{Conclusions}

Different searches for non-resonant HH production performed at CMS were presented. Four final states have been studied up to now and there is already a wide range of results obtained with data collected in 2016. The searches are not yet sensitive to the SM HH production but limits set from combination are more and more stringent; also BSM scenarios have been explored.

For the future we expect more: new final states will be studied and data collected in 2017 and 2018 will be analysed. Also data collected in Run 3 and High-Luminosity LHC will be included, thus many powerful results still have to come.

\section{References}

[1] Carvalho, A., Dall'Osso, M., Dorigo, T. et al, "Higgs pair production: choosing benchmarks with cluster analysis", JHEP 04 (2016) 126, 10.1007/JHEP04(2016)126

[2] CMS Collaboration, "The CMS Experiment at the CERN LHC”, JINST 3 (2008) S08004, 10.1088/1748-0221/3/08/S08004 


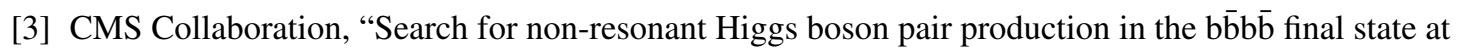
$\sqrt{s}=13$ TeV”, JHEP 04 (2019) 112, 10.1007/JHEP04(2019)112

[4] CMS Collaboration, "Search for resonant and non-resonant Higgs boson pair production in the bblnulnu final state in proton-proton collisions at $\sqrt{s}=13 \mathrm{TeV}$ ", JHEP 01 (2018) 054, 10.1007/JHEP01(2018)054

[5] CMS Collaboration, "Search for Higgs boson pair production in events with two bottom quarks and two tau leptons in proton-proton collisions at $\sqrt{s}=13$ TeV", Phys. Lett. B 778 (2018) 101 , 10.1016/j.physletb.2018.01.001

[6] CMS Collaboration, "Search for Higgs boson pair production in the $\gamma \gamma \mathrm{b} \bar{b}$ final state in pp collisions at $\sqrt{s}=13$ TeV”, Phys. Lett. B 788 (2019) 7, 10.1016/j.physletb.2018.10.056

[7] CMS Collaboration, "Combination of searches for Higgs boson pair production in proton-proton collisions at $\sqrt{s}=13$ TeV”, Phys. Rev. Lett. 122, 121803 (2019), 10.1103/PhysRevLett.122.121803 